\title{
Hypertensive cerebral hemorrhage with undetectable plasma VEGF after intravitreal injection of aflibercept for diabetic macular edema
}

\author{
Miwako Yoshimoto ${ }^{1}$, Nobuhiko Takeda ${ }^{2}$, Takayuki Yoshimoto², and Shun Matsumoto ${ }^{2}$ \\ ${ }^{1}$ Tokyo Teishin Hospital \\ ${ }^{2}$ Affiliation not available
}

September 11, 2020

\begin{abstract}
Anti-vascular endothelial growth factor (VEGF) therapy is widely used to maintain visual functioning in macular diseases including diabetic macular edema (DME). We report a case of a hypertensive cerebral hemorrhage even after intravitreal injections of aflibercept for DME in which the patient's plasma VEGF levels were below the detection limit.
\end{abstract}

\section{INTRODUCTION}

Vascular endothelial growth factor (VEGF) plays an essential role in normal and abnormal vasculogenesis and angiogenesis. ${ }^{1}$ VEGF stimulates endothelial cell proliferation, survival, and migration, which are necessary to form new blood vessels during embryonic development and wound healing and to develop new tumor vasculature in many cancers. ${ }^{1}$ Studies have shown that VEGF plays a central role in the pathogenesis of both cancers and eye diseases. ${ }^{1}$ The anti-VEGF monoclonal antibody, bevacizumab, was approved as a first-line treatment for metastatic colorectal cancer. ${ }^{2}$ Anti-VEGF therapy is also widely recommended to maintain visual functions in age-related macular degeneration, retinal vein occlusion, myopic choroidal neovascularization, and diabetic macular edema (DME). ${ }^{1}$ Large clinical studies ${ }^{3,4}$ have confirmed the safety of anti-VEGF therapy, but some meta-analyses ${ }^{5}$ indicate a high risk of systemic adverse events after repeated intravitreal anti-VEGF therapy for DME. This inconsistency may result from the study design strategy because large studies are often limited to patients with relatively fewer and minor complications and exclude patients at risk for the worst adverse events such as cerebrovascular and arteriothrombotic events. ${ }^{6}$ Because patients with DME generally exhibit systemic complications, investigating the direct adverse events of antiVEGF therapy for DME is difficult. Blockage of the VEGF pathway in cancer patients can cause adverse events such as hypertension, arterial thromboembolic events, cardiac dysfunction, proteinuria, renal toxicity, and compromised wound healing and tissue repair. ${ }^{7,8}$ Previous studies demonstrated that plasma VEGF levels were suppressed even after local intravitreal anti-VEGF therapy and continued to be decreased for several weeks. ${ }^{9,10}$ Other studies of age-related macular degeneration reported elevated blood pressure after intravitreal anti-VEGF therapy. ${ }^{11,12}$ However, evidence of a direct relationship between decreased plasma VEGF levels and adverse events, such as hypertension and resultant cerebral hemorrhaging, is lacking.

Here, we report a case of hypertensive cerebral hemorrhaging even after intravitreal injections of aflibercept for bilateral DME, in which the plasma VEGF levels remained below the detection limit before and after the stroke. We also present a comprehensive review of the relevant literature.

\section{CASE PRESENTATION}

A 70-year-old man with a 10-year history of diabetes was referred to our hospital to continue treatment for DME. At the patient's former hospital, he received one sub-Tenon's triamcinolone acetonide injection and intravitreal anti-VEGF injections 12 times in both eyes (4 times with $0.5 \mathrm{mg}$ ranibizumab and 7 times with 
$2 \mathrm{mg}$ aflibercept every 2-3 months in the right eye and once with aflibercept in the left eye) during the 2.5-year follow-up period. The last anti-VEGF therapy at the patient's former hospital was injected in the right eye in December 2016 and the left eye in July 2016.

At the patient's first visit to our hospital in January 2017, his HbA1c level was $6.3 \%$ with diet therapy only, and he was treated with donepezil hydrochloride for mild Alzheimer's dementia in the neurology department of Tokyo Takanawa Hospital. A dilated fundus examination showed mild nonproliferative diabetic retinopathy, with several small hemorrhages without macular edema, and his best corrected visual acuity was 1.0 in both eyes.

Five months later, he noticed blurred vision in both eyes, and a fundus examination showed significant macular edema. The central subfield macular thickness (CMT) on spectral-domain optical coherence tomography (SD-OCT) was 404/478 $\mu \mathrm{m}$ (right eye/left eye). In June 2017, for the first time at our hospital, the patient received 2-mg intravitreal aflibercept injections to both eyes 1 week apart (June 6 to the right eye and June 13 to the left eye). The anti-VEGF therapy was effective, and 1 month after the treatment, his CMT recovered to $277 / 321 \mu \mathrm{m}$, and his blurred vision disappeared. Three months after the first treatment at our hospital (September 1, 2017), significant macular edema relapsed in both eyes (CMT was 403/463 um; Fig. 1), and he desired continuous treatment for both eyes. In October 2017, he received intravitreal aflibercept injections in both eyes 1 week apart (October 3 to the right eye and October 10 to the left eye). One month after treatment (November 10), his blurred vision disappeared, and the CMT decreased to 280/315 $\mu \mathrm{m}$ (Fig. 2).

Upon initiating the intravitreal injection in the operating room, we measured his blood pressure at 141/69 $\mathrm{mmHg}$ (October 3) and 135/70 $\mathrm{mmHg}$ (October 10) without blood-pressure-lowering drugs (Fig. 3A). However, on November 12, just 2 days after a follow-up visit to our hospital, he complained of a severe headache and walked to the emergency department at Tokyo Takanawa Hospital. He complained of a severe headache on the right back side of his head and visual disturbance of the left visual field with no other systemic neuropathological symptoms. His blood pressure was elevated at 195/108 $\mathrm{mmHg}$, and computed tomography (CT) of the brain revealed cerebral hemorrhaging of the right occipital lobe (Fig. 4). The patient was treated orally with $40 \mathrm{mg}$ /day telmisartan to lower his blood pressure, which promptly dropped to $130 / 60 \mathrm{mmHg}$ within the same day. Magnetic resonance angiography demonstrated no vascular anomalies (data not shown), and he was diagnosed with hypertensive cerebral hemorrhage of the occipital lobe. Ten days later, he was discharged from Tokyo Takanawa Hospital, and 20 days after discharge he visited our hospital to receive a follow-up ophthalmological examination. Two months after the stroke (January 5, 2018), visual field tests with Goldmann perimetry at our hospital showed no apparent left homonymous hemianopsia (Fig. 5). One year after the cerebrovascular stroke, the telmisartan was discontinued because his blood pressure had stabilized at a normal level, and after that it kept at around 110/60 mmHg.

The patient received no anti-VEGF therapy for 4 months prior to October 3 because his macular edema remained stable. We determined his plasma VEGF levels before and after the intravitreal anti-VEGF injections (October 2017) via specific enzyme-linked immunosorbent assay (SRL, Tokyo, Japan; Fig. 3B). The patient's plasma VEGF level was $28 \mathrm{pg} / \mathrm{ml}$ on October 3, just before the intravitreal injection of aflibercept to the right eye. However, the level rapidly decreased to below the detection limit $(<20 \mathrm{pg} / \mathrm{ml})$ on October 10, just before the injection to left eye. Of note, the levels on November 10 (2 days before the cerebral hemorrhage) and December 20 were also below the detection limit. On January 25, 2018, we confirmed that his plasma VEGF level had recovered to $41 \mathrm{pg} / \mathrm{ml}$. Thereafter, we continued to follow the patient for DME and diabetic retinopathy, and no cerebral hemorrhaging recurred.

\section{DISCUSSION}

This is the first report showing a case who experienced a hypertensive cerebral hemorrhage after intravitreal injections of aflibercept for bilateral DME in which the patient's plasma VEGF levels were below the detection limit before and after his stroke. The decreased plasma VEGF levels is highly considered to have a causal relationship with the hypertensive cerebral hemorrhage. This is because VEGF plays an essential role 
in normal and abnormal vasculogenesis and angiogenesis; VEGF induces new blood vessel formation by increasing the capillary and arteriolar density and releases nitric oxide and prostaglandins via endothelial cells to promote vasodilation. ${ }^{1,13}$ In the absence of VEGF, normal blood vessel formation by endothelial cells is impaired and weakened and thus more susceptible to hypertension and resultant hemorrhaging. Although this report represents only one case, clinicians should keep in mind that adverse events, including hypertension and resultant cerebral hemorrhaging, can occur if the plasma VEGF levels fall below the detection limit during anti-VEGF therapy for DME.

Large clinical trials have demonstrated the safety of intravitreal anti-VEGF agents, but longer term, repeated injections of anti-VEGF agents may increase the risk of systemic adverse events. ${ }^{5,8}$ When bevacizumab was intravenously injected to treat solid tumors, several adverse events were reported because of the diminished VEGF activity such as hypertension, arterial thromboembolic events, myocardial infraction, and stroke. ${ }^{7,8}$ Plasma VEGF levels that systemically fall below the detection limits even due to local intravitreal injections of anti-VEGF agents pose risks similar to those seen in cancer patients. ${ }^{14}$ The three most common antiVEGF agents are bevacizumab, aflibercept, and ranibizumab. Bevacizumab is a recombinant humanized monoclonal anti-VEGF antibody containing an Fc domain, through which neonatal Fc receptor recycling may prolong the half-life to approximately 20 days after intravenous injection. ${ }^{15}$ Aflibercept is a soluble fusion protein consisting of ligand-binding domains of the extracellular region of VEGF receptors 1 and 2 and the Fc domain of human IgG1. Its half-life in the plasma was reported to be 5-6 days after intravenous injection. ${ }^{9}$ Ranibizumab is a monoclonal antibody Fab fragment created from the same parental mouse antibody against VEGF-A as that of bevacizumab but without the Fc domain. Therefore, ranibizumab is more rapidly cleared from the circulation, with a half-life of just 2 hours. ${ }^{16}$ Consistent with these molecular properties, bevacizumab and aflibercept were reported to greatly reduce plasma VEGF levels at 1 and 4 weeks after a single intravitreal injection, whereas ranibizumab had no significant effect. ${ }^{10}$ However, ranibizumab showed a possible risk of cerebrovascular accidents when used monthly for 2 years. ${ }^{5}$ Importantly, intraocular VEGF levels were recently correlated with retinal neovascularization and consequently DME resolution, but plasma VEGF levels were unrelated to the DME severity or diabetic retinopathy. ${ }^{10,17,18}$ Therefore, decreased plasma VEGF levels are not necessary to treat DME, and the total amounts of anti-VEGF agents should be minimized to avoid prolonged decreases in plasma VEGF levels and possible resultant hypertensive accidents in DME patients.

Risk factors of DME are high hemoglobin A1c levels and a long duration of diabetes, ${ }^{19}$ and many patients with DME have cardiovascular and cerebrovascular complications. Intravitreal anti-VEGF therapy improves visual outcomes and decreases macular edema, but the response is more gradual than that of other retinal diseases. ${ }^{20}$ Patients with diabetes require more intravitreal injections of anti-VEGF medications to attain maximal improvement of visual outcomes. These injections must be given more often because DME is often binocular. Thus, to treat DME, clinicians should attempt to reduce the number of repeated antiVEGF injections by combining them with other therapies (direct photocoagulation of microaneurysms or sub-Tenon's triamcinolone acetonide injection and vitrectomy) as well as systemic improvement of diabetes control and lipid metabolism. If anti-VEGF therapy must be frequently repeated, changes in blood pressure and other systemic pathological objective conditions should be monitored at every treatment in addition to the patient's self-reported health status, especially in concomitant binocular treatment. If the technique to determine plasma VEGF levels would so advance that they would be more easily and rapidly measured even just before an operation, anti-VEGF therapy for DME could become safer, and a more effective treatment schedule could be determined. These approaches will lead to better and safer vision and life quality for patients with diabetes.

\section{CONCLUSION}

Hypertension and resultant cerebral hemorrhaging can occur as adverse events in patients with DME when plasma VEGF levels systemically fall below the detection limit after even local injection of anti-VEGF agents into the vitreous cavity. Therefore, ophthalmologists should be aware of increases in blood pressure even after intravitreal injections of an anti-VEGF drug, and plasma VEGF levels should be monitored during the 
treatment just in case.

\section{CONFLISCT OF INTEREST}

None declared.

\section{AUTHOR CONTRIBUTIONS}

MY: analyzed and interpreted the patient's data, produced the images used in the manuscript, and wrote the manuscript. NT: analyzed and interpreted the patient's data. TY and SM: reviewed the manuscript. All authors read and approved the final manuscript.

\section{ETHICAL APPROVAL}

This study protocol was approved by the Ethics Committee of Tokyo Teishin Hospital (No. 1039). The Declaration of Helsinki was followed in this case report.

\section{CONSENT STATEMENT}

Written informed consent was obtained from the patient, including permission to publish case details and photographs.

\section{FUNDING INFORMATION}

The authors received no specific funding for this work.

\section{REFERENCES}

1. Apte RS, Chen DS, Ferrara N. VEGF in Signaling and Disease: Beyond Discovery and Development. Cell. 2019;176(6):1248-1264.

2. Ranieri G, Patruno R, Ruggieri E, Montemurro S, Valerio P, Ribatti D. Vascular endothelial growth factor (VEGF) as a target of bevacizumab in cancer: from the biology to the clinic. Curr Med Chem.2006;13(16):1845-1857.

3. Virgili G, Parravano M, Evans JR, Gordon I, Lucenteforte E. Anti-vascular endothelial growth factor for diabetic macular oedema: a network meta-analysis. Cochrane Database Syst Rev.2017;6:CD007419.

4. Maloney MH, Schilz SR, Herrin J, Sangaralingham LR, Shah ND, Barkmeier AJ. Risk of Systemic Adverse Events Associated with Intravitreal Anti-VEGF Therapy for Diabetic Macular Edema in Routine Clinical Practice. Ophthalmology. 2019;126(7):1007-1015.

5. Avery RL, Gordon GM. Systemic Safety of Prolonged Monthly Anti-Vascular Endothelial Growth Factor Therapy for Diabetic Macular Edema: A Systematic Review and Meta-analysis. JAMA Ophthalmol.2016;134(1):21-29.

6. Kawasaki R, Tanaka S, Tanaka S, et al. Risk of cardiovascular diseases is increased even with mild diabetic retinopathy: the Japan Diabetes Complications Study. Ophthalmology. 2013;120(3):574-582.

7. Chen HX, Cleck JN. Adverse effects of anticancer agents that target the VEGF pathway. Nat Rev Clin Oncol. 2009;6(8):465-477.

8. Falavarjani KG, Nguyen QD. Adverse events and complications associated with intravitreal injection of anti-VEGF agents: a review of literature. Eye (Lond). 2013;27(7):787-794.

9. Avery RL, Castellarin AA, Steinle NC, et al. Systemic Pharmacokinetics and Pharmacodynamics of Intravitreal Aflibercept, Bevacizumab, and Ranibizumab. Retina. 2017;37(10):1847-1858.

10. Hirano T, Toriyama Y, Iesato Y, Imai A, Murata T. Changes in Plasma Vascular Endothelial Growth Factor Level after Intravitreal Injection of Bevacizumab, Aflibercept, or Ranibizumab for Diabetic Macular Edema.Retina. 2018;38(9):1801-1808. 
11. Rasier R, Artunay O, Yuzbasioglu E, Sengul A, Bahcecioglu H. The effect of intravitreal bevacizumab (avastin) administration on systemic hypertension. Eye (Lond). 2009;23(8):1714-1718.

12. Hanna RM, Lopez EA, Hasnain H, et al. Three patients with injection of intravitreal vascular endothelial growth factor inhibitors and subsequent exacerbation of chronic proteinuria and hypertension. Clin Kidney J. 2019;12(1):92-100.

13. Verheul HM, Pinedo HM. Possible molecular mechanisms involved in the toxicity of angiogenesis inhibition. Nat Rev Cancer.2007;7(6):475-485.

14. Avery RL, Castellarin AA, Steinle NC, et al. Systemic pharmacokinetics following intravitreal injections of ranibizumab, bevacizumab or aflibercept in patients with neovascular AMD. Br J Ophthalmol. 2014;98(12):1636-1641.

15. Krohne TU, Eter N, Holz FG, Meyer CH. Intraocular pharmacokinetics of bevacizumab after a single intravitreal injection in humans. Am J Ophthalmol. 2008;146(4):508-512.

16. Xu L, Lu T, Tuomi L, et al. Pharmacokinetics of ranibizumab in patients with neovascular age-related macular degeneration: a population approach. Invest Ophthalmol Vis Sci. 2013;54(3):1616-1624.

17. Funatsu H, Yamashita H, Noma H, et al. Aqueous humor levels of cytokines are related to vitreous levels and progression of diabetic retinopathy in diabetic patients. Graefes Arch Clin Exp Ophthalmol. $2005 ; 243(1): 3-8$.

18. Dong N, Xu B, Chu L, Tang X. Study of 27 Aqueous Humor Cytokines in Type 2 Diabetic Patients with or without Macular Edema. PLoS One.2015;10(4):e0125329.

19. Acan D, Calan M, Er D, et al. The prevalence and systemic risk factors of diabetic macular edema: a cross-sectional study from Turkey.BMC Ophthalmol. 2018;18(1):91.

20. Ziemssen F, Schlottman PG, Lim JI, Agostini H, Lang GE, Bandello F. Initiation of intravitreal aflibercept injection treatment in patients with diabetic macular edema: a review of VIVID-DME and VISTA-DME data.Int $J$ Retina Vitreous. 2016;2:16.

\section{FIGURE LEGENDS}

FIGURE 1 SD-OCT findings from September 1, 2017. A: right eye thickness map; B: left eye thickness map; C: right eye line scan; D: left eye line scan. Upper figure is the horizontal scan; lower figure is the vertical scan of the macula. Three months after anti-VEGF therapy in both eyes, the concomitant binocular DME relapsed. Yellow arrows indicate cystoid macular edema.

FIGURE 2 SD-OCT findings from November 10, 2017 (1 month after treatment). A, B, C, and D are the same as in Figure 1. Anti-VEGF therapy was effective, and the binocular DME disappeared entirely. Yellow arrows indicate disappearance of the cystoid macular edema.

FIGURE 3 Systolic and diastolic blood pressure (A) and plasma VEGF levels (B) of the patient before and after binocular anti-VEGF therapies 1 week apart. More than 2 months after anti-VEGF therapy in both eyes, the plasma VEGF levels fell below the detection limit $(<20 \mathrm{pg} / \mathrm{ml})$. On November 12, 2017, cerebral hemorrhaging occurred due to acute hypertension, and the patient was treated orally with $40 \mathrm{mg} /$ day telmisartan to lower his blood pressure, which promptly dropped to a normal level within the same day.

FIGURE 4 Computed tomography (CT) of the brain on November 12, 2017 (A) and February 14, 2018 (B), and T2* magnetic resonance imaging (MRI) of the brain on November 15, 2017 (C), and July 24, 2018 (D). High density on the CT (A, yellow arrowhead) and low intensity on the $\mathrm{T} 2^{*} \mathrm{MRI}$ (C, yellow arrowhead) revealed cerebral hemorrhaging of the right occipital lobe. Three months after the stroke, the high-density area disappeared, and the small low-density area remained (B, yellow arrowhead) on the brain CT and the $\mathrm{T}^{*}$ MRI. The low-intensity lesion decreased in size 8 months later ( $\mathrm{D}$, yellow arrowheads). 
FIGURE 5 Goldmann perimetry of both eyes on January 5, 2018 (2 months after the cerebral hemorrhage). Goldmann perimetry showed no apparent visual field abnormalities.

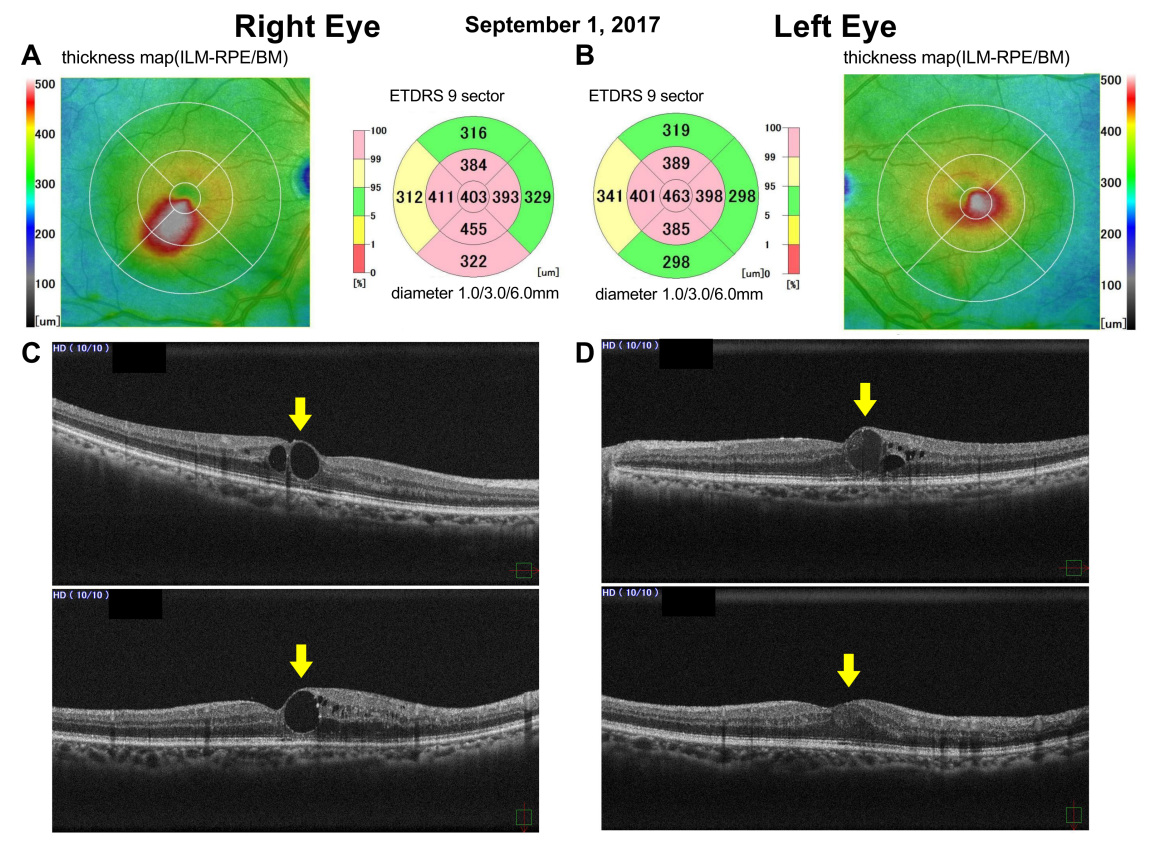

Figure 1. Yoshimoto et al.
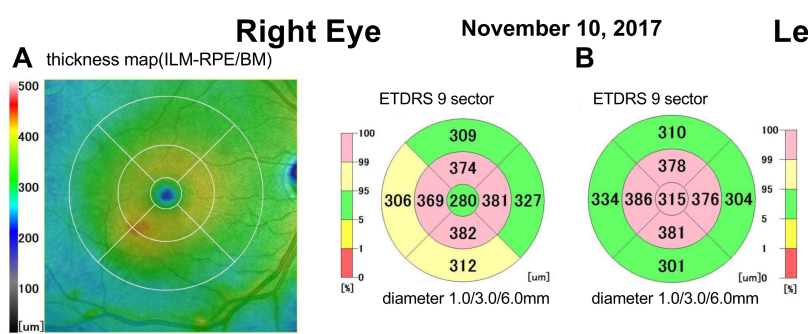

Left Eye
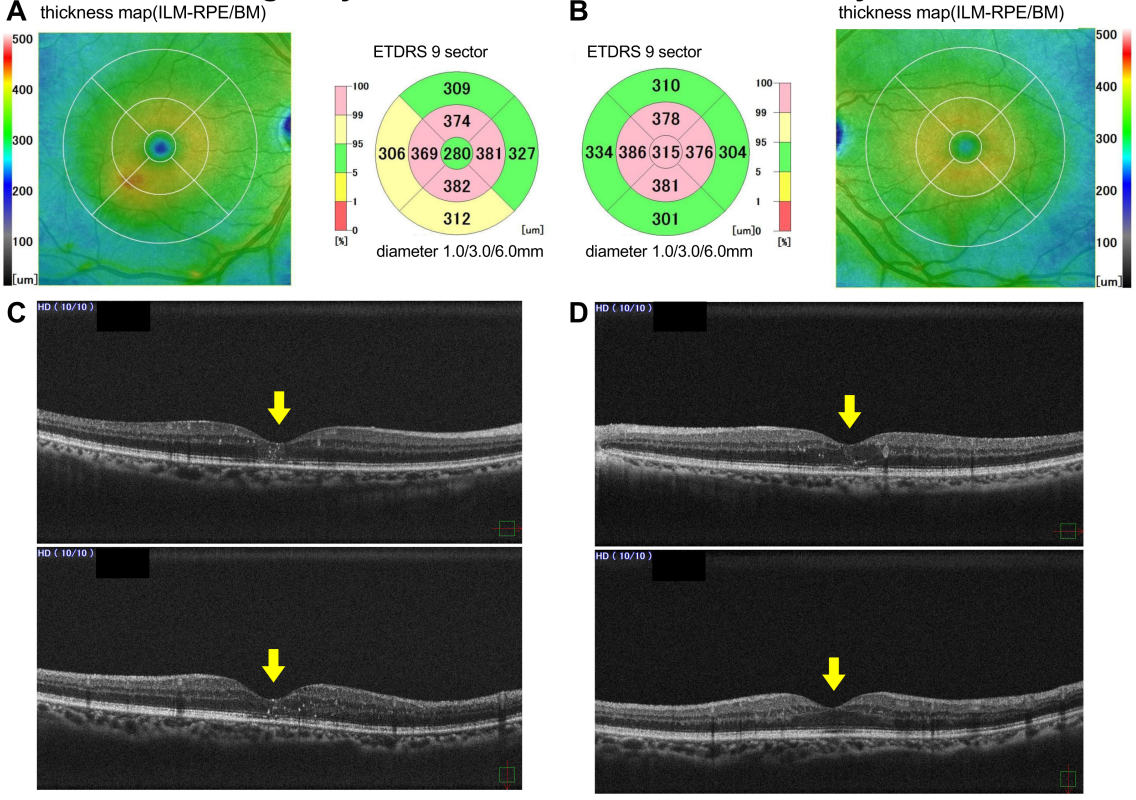

Figure 2. Yoshimoto et al. 

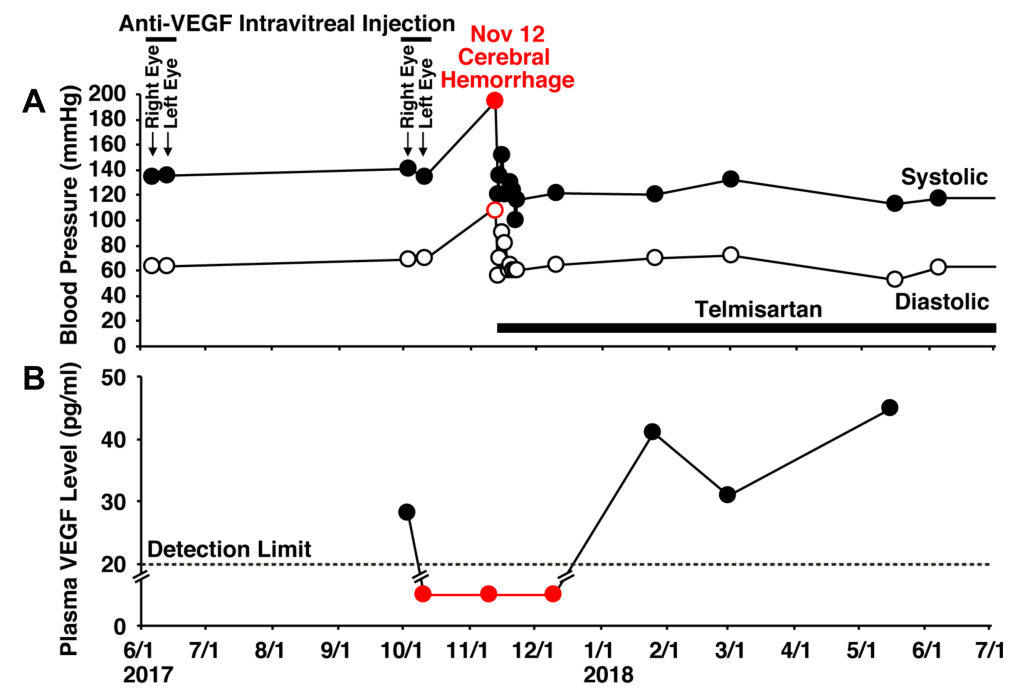

Figure 3. Yoshimoto et al.
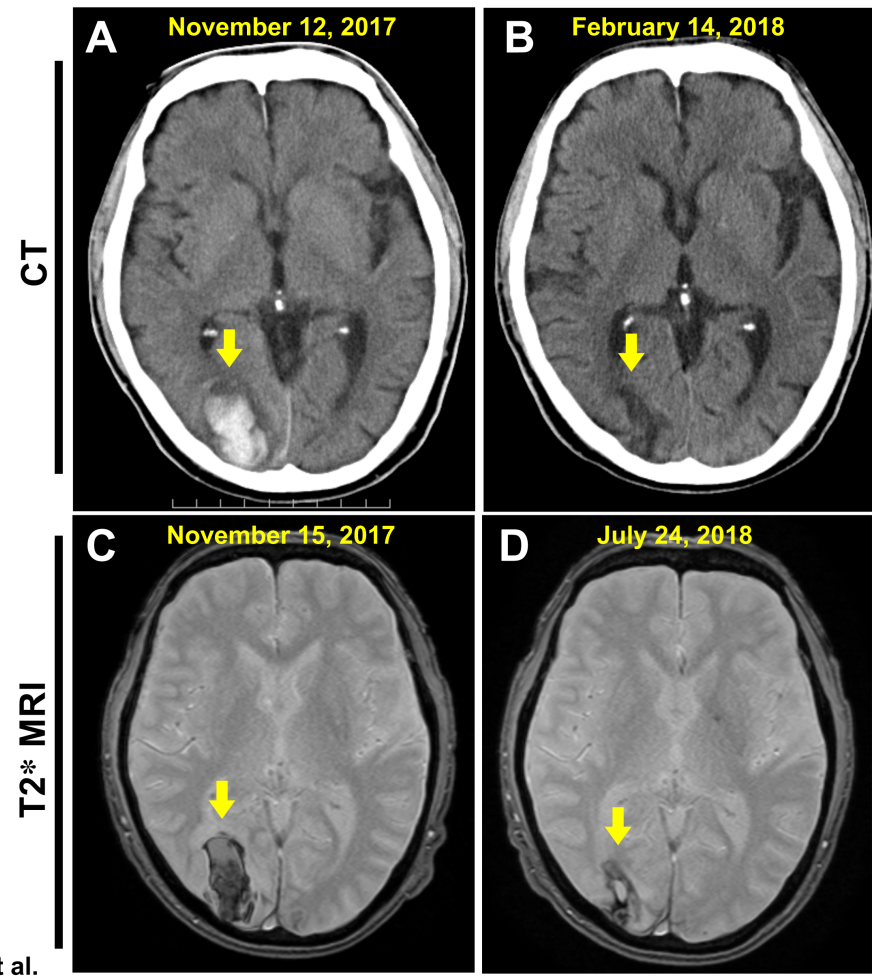

Figure 4. Yoshimoto et al. 


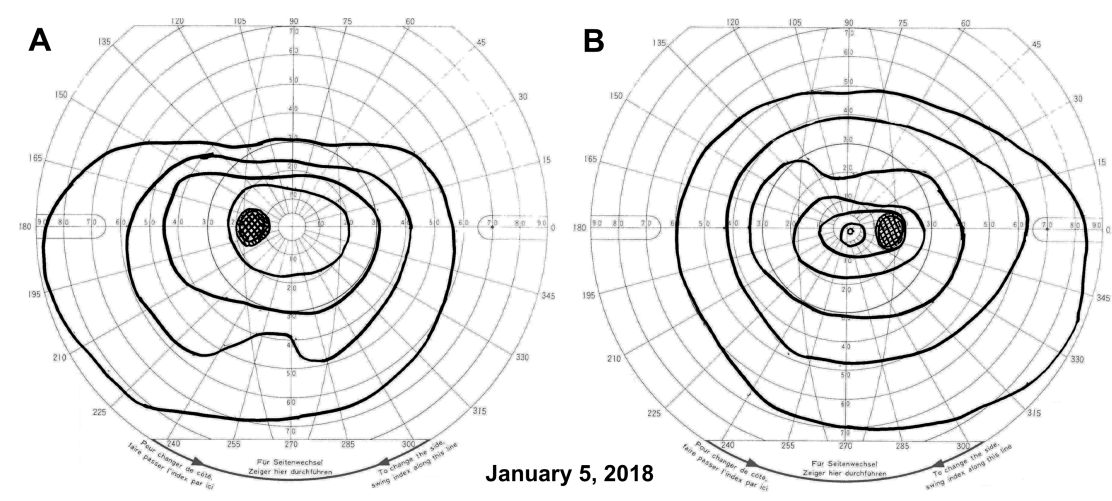

Figure 5. Yoshimoto et al. 